\title{
Designing an asynchronous multi-channel media access control protocol based on service quality for wireless sensor networks
}

\author{
Mahboobe Irandegani and Mehdi Bagherizadeh* \\ Department of Computer Engineering, Rafsanjan Branch, Islamic Azad University, Rafsanjan, Iran
}

Received: 26-June-2017; Revised: 24-August-2017; Accepted: 29-August-2017

(C)2017 ACCENTS

\begin{abstract}
Recent developments in the field of telecommunication have provided the ability to design and manufacture wireless sensor networks (WSNs) with low power consumption, small size and affordable prices. The structure of these networks is such that sensor nodes are placed in inaccessible places, because of that it is almost impossible to charge or replace their batteries. Due to the limited resources of these nodes, the main challenge in these networks is to manage energy consumption and increase their lifetime. Media access control protocols (MACs) are introduced in the lower layer of the network, and their main function is to provide access to a common media in order to avoid collisions between different nodes. In this paper, an asynchronous multi-channel media access control protocol was introduced to configure the sleep and wake-up time of the nodes as well as set the meeting of the nodes in order to exchange data. This protocol makes it possible to use multiple channels for sending and receiving frames without creating synchronization overhead. And as a result, it reduces energy consumption and increases packet delivery rates on the network. The evaluation results of the proposed protocol show that the proposed method works better in energy consumption, operational power, delay and the ratio of packets to the destination, package rate and send multiple data steps compared to other MAC-based methods.
\end{abstract}

\section{Keywords}

Wireless sensor networks (WSNs), Media access control protocol (MAC), Non-continuous multi-channel media access control protocol, Energy consumption and lifetime.

\section{Introduction}

Wireless sensor networks (WSNs) are one of a variety of distributed systems that have been considered by many researchers in recent years and have been used in many places, such as military environments, banks, airports and other places in order to be used in security systems. Wireless sensor networks are a kind of multi-hub network, which consist of a large number of low-cost sensor nodes. The nodes are wirelessly connected and are capable of communication and processing [1, 2]. Sensor nodes are small and intelligent devices with wireless capability that can receive and process various data such as light, sound, temperature, etc., and eventually transmit to other nodes. The main task of these nodes is to collect data at regular intervals. After collecting data, all nodes send the data for further processing to a central node which is called a sink.

\footnotetext{
*Author for correspondence

This work was supported in part by Department of Computer Engineering, Rafsanjan Branch, Islamic Azad University, Rafsanjan, Iran.
}

The sink node sends the data directly or indirectly to a central station for final management after initial processing [3]. Although each sensor has little ability, but the combination of hundreds of small sensors offers new features. In fact, the power of wireless sensor networks is the capable of employing a large number of small nodes that can be organized and can be used in many cases, such as routing, monitoring environmental conditions, monitoring the health of structures or equipment of a system [4]. The application range of wireless sensor networks is vast and covers agribusiness, medical, industrial and trooper applications. One of the most common uses of this technology is monitoring a remote environment. For example, the leakage of a chemical factory in the factory environment or the nuclear, chemical and microbial attacks of the enemy can be monitored by hundreds of sensors that automatically form a wireless network and get promptly notified to the center when problems occur [5].

There are various challenges of this type of network and the problem of resource constraints is the most important one [6]. There are different factors that lead to energy dissipation in sensor nodes. The most 
important factors that have been addressed in in the previous studies in order to manage energy consumption are as follows: collision, overhearing, protocol overhead, idle listening, and over meeting [7].

In most research related to wireless sensor networks, routing algorithms, aggregation methods, and data redundancy or media access control protocols have been used to reduce node energy consumption [8]. In the remainder of this paper, section 2 introduces several media access protocols. Section 3 describes the proposed MAC protocol by expressing the features. The fourth section deals with the implementation, structure and network model and in section 5, the results obtained from the implementation of the proposed method in the energy consumption criterion and several other criteria are presented. Section 6 provides a general conclusion.

\section{Media access control protocol formatting}

Media access control protocols are introduced in the lower layers and their main function is to create access to a common media in order to avoid collisions between different nodes. In networks with shared media, media access control is essential for successful network operations. In addition to the core functionality, the media access control protocol must incorporate other factors in its design to enhance the network's performance and provide appropriate services with different applications. In wireless sensor networks, these factors, mainly include energy efficiency, scalability, adaptability, channel efficiency, delays, throughput and fair sharing. But energy efficiency is more valuable [9]. Considering that the main goal of media access control protocols is the reduction of energy consumption, so the most important step is to identify the cases that lead to energy dissipation. Then the protocol seeks to improve these issues.

In order to avoid collisions, media access control protocols are usually classified into competitive and non-competitive categories. In controlling access to competitive media, all nodes use a common medium to challenge for data transfer. Therefore, collisions may occur during the competition process. In order to prevent collisions, the media access control protocol can be used to access the shared channel through potential coordinates. ALOHA protocol and carriersense multiple access (CSMA) protocol are the most common examples of competitive media access control protocols. In the access control of a media without competition, the common media is divided into a number of sub channels in terms of time, frequency, or orthogonal pseudo noise code. Each node occupies a sub-channel. Allocating distinct channels to different nodes provides access to common media without interfering. Therefore, this method avoids the collision of different nodes effectively. The most common examples of noncompetition media access control protocols are timedivision multiple access (TDMA), frequency division multiple access (FDMA) and code division multiple access (CDMA). TDMA divides the common channel into constant time intervals and configures these intervals in a way that they are repeated periodically. In this method, a time interval is assigned to each node to carry out in the same framework.

\subsection{Introducing some media access control protocol}

Moreover, several media access control protocols have been introduced and each of them is trying to resolve the challenges in wireless sensor networks. Some of these protocols are introduced and evaluated in the following.

\section{S-MAC protocol}

The sensor MAC (S-MAC) protocol is an energy efficient MAC protocol specifically designed for wireless sensor networks [10]. S-MAC is designed for the sensor network, where most of the communication between nodes occurs in peer-to-peer mode and there is no central station. Therefore, its applications have long idle periods. S-MAC can tolerate the latency of sending messages. The main goal of designing S-MAC protocol is to increase energy efficiency, while maintaining scalability and collision avoidance. In order to achieve this goal, the S-MAC protocol tries to reduce energy consumption from all sources that cause inefficient use of energy. In contrast, there is the possibility of degrading performance in fair sharing and delays in each folder. This is done by combining several effective protocol mechanisms in a competitive MAC protocol which is based on the IEEE 802.11 standard. These mechanisms include: listening and sleeping periodically, avoiding collisions, coordinated synchronization and passing the message [10].

\section{WiseMAC Protocol}

WiseMAC is an efficient energy-efficient MAC protocol, which is provided by El-Hoiydi et al. for multicast and infrastructure networks [11]. In order to enhance energy efficiency, the protocol combines 
CSMA with pre-synchronized sampling to reduce idle time listening. In the preliminary sampling technique, all the nodes in the network are sampled in the same constant period of the media, but the starting point of sampling timing is independent. If the media is busy, it will continue to listen until it receives the data packet or the media becomes idle again. At the sender node, the initial wakeup time that is as long as the sampling period is sent before sending each packet to change the receiver node. In low traffic, this technique consumes a small amount of power, but the initial length of the overhead causes the high initial power consumption in sending and receiving.

\section{CSMA based method}

Woo and Culler has introduced a CSMA-based MAC protocol with an adaptive control rate [12]. This protocol incorporates CSMA with adaptive rate control mechanism. The protocol accepts a particular network scenario in which the base station receives data from all sensors in the desired field, and it generates desired applications and high and intermittent traffic. This is done with the goal of energy efficiency and fair allocation of bandwidth for all nodes in a multi-hop network. It has been proved that the CSMA-based MAC protocol is more effective for achieving fairer bandwidth allocations, while at the same time it is energy-efficient with low and high network traffic. However, because it is based on CSMA, it may suffer from overhead control and hidden station problems.

\section{T-MAC protocol}

Timeout MAC (T-MAC) is an energy efficient protocol that has been introduced by Dam and Landendoen for wireless sensor networks. The initial idea of T-MAC was to reduce the duration of listening in the idle state by using a dynamic workflow. Additionally, in this protocol all messages are transmitted in explosive mode with variable size in active periods, and nodes go to sleep state in intervals between active periods. In order to maintain an optimal active period under varying traffic loads, if nothing has been heard, T-MAC dynamically determines the duration of the active period by reaching the time limit [13].

\section{D-MAC protocol}

Lu et al. introduced a low latency energy efficient dynamic MAC (D-MAC) protocol for data collection in wireless sensor networks [14]. This protocol is presented to address the issue of interfering leading forwarding of data in multi-hop data delivery and its main objective is to achieve both the efficiency of the power and the low latency. In D-MAC, a scheduler is used to wake up in order to continue the leading forwarding of data in multi-hop path. In wireless sensor networks, primary traffic relates to data gathering from sensor nodes to the sink. Delivery routes from multiple sources to a sink form a data aggregation tree, in which the data flows are unidirectional and all the nodes (except the sink) receive packets and send to the next jump.

\section{DS-MAC Protocol}

Demand sleep Mac (DS-MAC) is an S-MAC-based protocol with dynamic workflow. This protocol is designed to compromise energy consumptions and delays without creating too much overhead [15]. In DS-MAC, each sensor node assumes that all of its work is defined in S-MAC, and each receiver node remembers its path, energy consumption, and intermediate delay. To achieve the desired agreement, each node attempts to adjust the wake-up and sleep cycle time based on the current level of energy consumption and the average delay it has saved. Average delay is used as an estimate for current traffic conditions and a basic parameter for the receiver. This protocol reduces the high latency problem by placing the S-MAC under high traffic conditions so that it achieves high power efficiency under low traffic loads.

\section{MS-MAC protocol}

MAC protocol for sensor networks (MS-MAC) is a MAC protocol that is aware of dynamic mobility. MS-MAC is provided to address the mobility issue in mobile sensor applications such as patient intelligence assistant and rare animal monitoring [16]. This protocol uses any changes made at the signal levels received from intermittent SYNC messages as an indication of mobility and, if necessary, targets the mobility management mechanism. The mechanism dynamically adjusts the frequency of actions related to managing mobility based on the presence of moving nodes and their speed up. With such a mechanism for estimating mobility, MS-MAC is economical in terms of energy consumption for stationary situations while maintains a certain level of network performance in positions with mobile sensor nodes.

\section{EM-MAC protocol}

Efficient multichannel MAC (EM-MAC) protocol belongs to the asynchronous duty cycled MAC protocols that uses multiple available radio channels [5]. Contrary to existing multi-channel protocols, 
EM-MAC specifies the channel's dynamic channel dynamically. Channel selection depends on its features. EM-MAC prevents heavy traffic on one channel by creating an effective efficiency method. This efficient energy efficiency approach is achieved by precisely predicting the channel and the wake up time of the receiving node. Each time a node wakes up; the time is determined by a pseudorandom function. Of course channels with high traffic are detected and selecting them will be prevented. The sender is aware of the receiver's pseudorandom function, the wake-up time and the wake up channel of each receiver for an effective communication.

\section{MIMO protocol}

Multiple-input multiple-output (MIMO) method increases the network energy efficiency if configured well [17]. A new media access protocol has been introduced for clustered wireless sensor networks that utilize multi-channel benefits and the MIMO collaboration method to increase network efficiency. The cluster heads select a number of nodes as an aid by the MIMO method for the time when traffic received from other clusters is high. For interconnected clusters, different channels are assigned to adjacent clusters and for the connection between the clusters, the MIMO collaborator connections are planned for energy efficiency and simultaneous transmission by assigning different channels is possible.

\section{AS-MAC protocol}

The asynchronous scheduled MAC (AS-MAC) protocol is an asynchronous single-channel media access control protocol. The AS-MAC protocol operation consists of initialization step and a periodic listening and sleep step. When a node joins the network, this node starts up with the initialization phase. This phase includes exploration channels, wake scheduling, main channel and announcement. This node is listening to the hi-res message, in order to collect channel information and node scheduling, for longer than the hi-res period in each channel. In AS-MAC, each node wakes up alternately on its asynchronous scheduling with its neighbors, and performs low-power listening operation or low power listening (LPL) each time it wakes up.

\section{Proposed protocol}

The overall framework of the proposed protocol and its stages are roughly analogous to the AS-MAC protocol, But since the AS-MAC protocol is an asynchronous single-channel media access control protocol, we extend it to a multi-channel architecture. Figure 1 shows the overall structure of the proposed protocol.

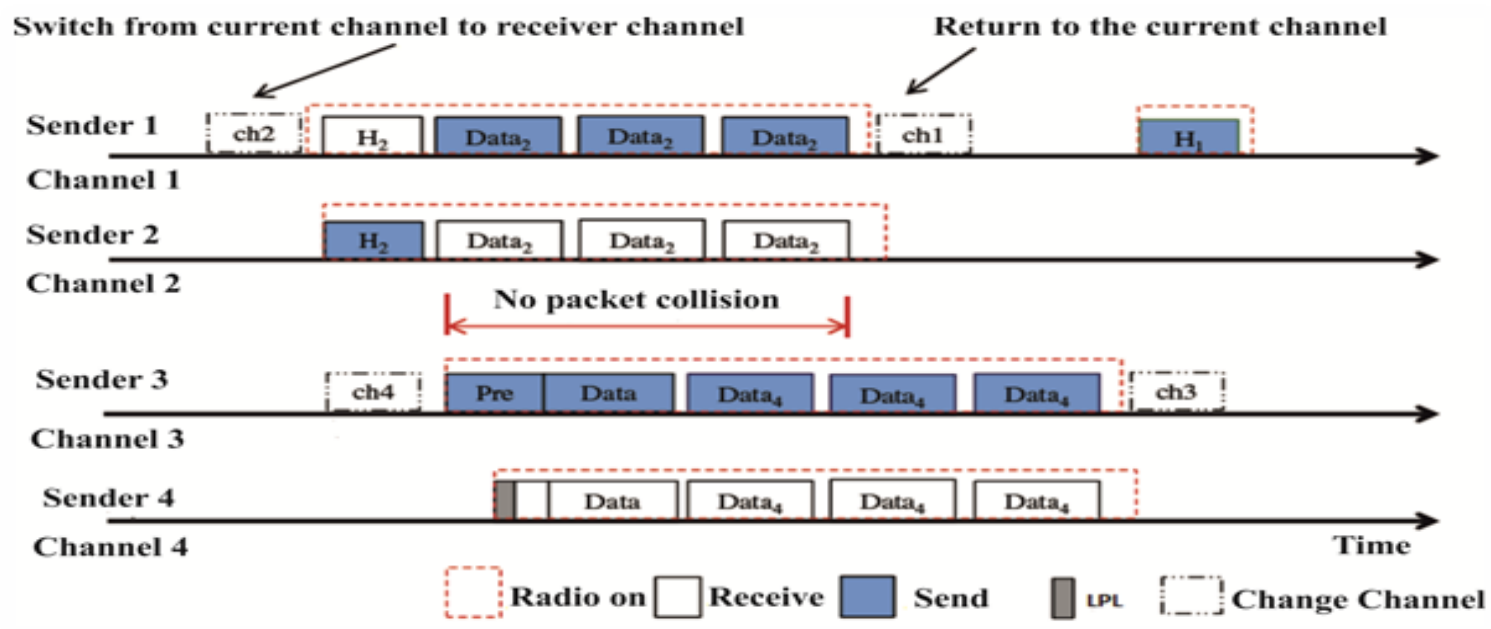

Figure 1 The overall structure of the proposed protocol

\subsection{High traffic support in the proposed method} In order to protect the high traffic load of the network, the mode of sending packets is activated in a row using extra time after receiving and sending them. As shown in Figure 2, after transmitting a data packet, the transmitter remains awake for a traffic- adaptive synchronous (TAS) time period, and in order to enable the upper layers to request additional packets, the packets are sent in a row. After receiving the data packets, the receiver will stay awake for a short time $\left(\mathrm{T}_{\mathrm{AR}}\right)$ until it can receive the packets after receiving the previous packets. 


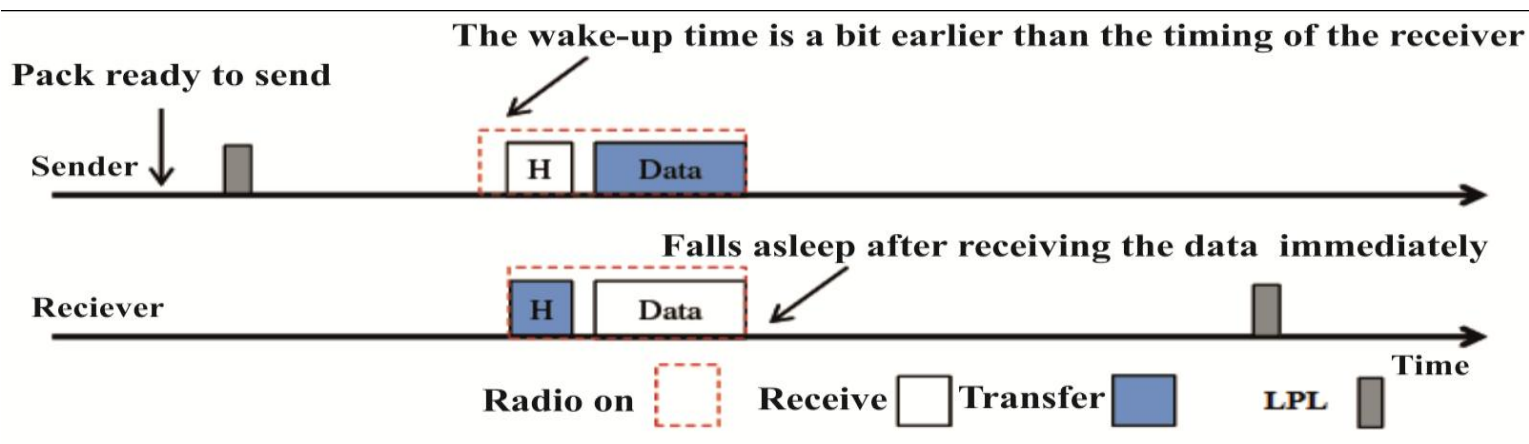

Figure 2 Support high traffic by additional wake-up time

If an error occurs during the sending of data from the sender's side, the receiver cannot receive packets in a row. The reason for not receiving consecutive packets is that if the receiver fails to receive the additional data packet during the $\mathrm{T}_{\mathrm{AR}}$, it will return to sleep state. In the proposed method, for the reliable transmission of packets and preventing the loss of consecutive packets, collision identification and resend method from [18] is extended. So that if a node receives a packet correctly it sends an ACK packet, and if a node sends a packet and does not receive the corresponding ACK packet, it will resend the packet at the time of the next awakening. In the detection of collision and retransmission method, if a node receives a packet correctly it will send an ACK packet and then waits for an ACK packet at the time of TAS2 (for example, a little longer than the sending time, the processing time that the receiver processes the data, the processing time that receiver make ACK packet and the sending time that the receiver sends the ACK packet.) If the sender receives an ACK packet at the time of TAS2, it can send an additional packet, Otherwise it will go to sleep and delay the sending of the packet to the next awaiting time of the receiver.

\subsection{Multiple channel support}

Due to energy consumption and cost constraints, many sensors have been equipped with a transceiver. Therefore, the proposed method is designed for nodes with a half-duplex transceiver. With regard to Figure 1 , each node has its own main channel in this method, which is selected during the initialization phase. Each node sends a hello message at each time. Hello message includes a Src (sender's mac address of source node) a wake-up time, a hello time period, and a main channel. Every node that receives hello message saves its information on hello message when the sign of starting message detected in its neighbours table. The hello message format is shown in Figure 3.

\begin{tabular}{|l|l|l|l|l|l|l|l|}
\hline Preamble & Length & Type & Src & $\begin{array}{l}\text { Wakeup } \\
\text { Interval }\end{array}$ & $\begin{array}{l}\text { Hello } \\
\text { Interval }\end{array}$ & $\begin{array}{l}\text { Home } \\
\text { Channel }\end{array}$ & CRC \\
\hline
\end{tabular}

Figure 3 Hello message format in the proposed method

If a node has packets for sending, the sender will set the remaining time to wake up the receiver along with the main channel in which the receiver will wake up. Because the sender has to change its channel to the receiver, main channel, the time for changing the $t_{\text {switch }}$ channel must be calculated. This value is calculated from the equation (1).

$$
\begin{aligned}
\operatorname{Tremain}(r)= & (\text { Iwakeup }(r) \\
& -\operatorname{tcurrent}-\operatorname{tirt}(\mathrm{r}) / \text { Iwakeup }(\mathrm{r}) \\
& - \text { twakeup }- \text { thbf }- \text { tswitch }
\end{aligned}
$$

Due to the fact that the main channel of each node is selected during the initialization phase and each node manages its neighbor scheduling with the main channels, the proposed method does not need to coordinate between the nodes throughout different channels and has the available bandwidth for sending data.

\subsection{Initialization step}

When a node joins the network, this node starts up with the initialization phase. This phase includes exploration channels, wake scheduling, main channel and announcement. This node is listening to the hello message, in order to collect channel information and node scheduling, for longer than the hello message period in each channel. According to this 
information, the channel that is least used is selected as the main channel of the new node. Given that it is impossible for a node to send and receive at one time using one transceiver, a unique scheduler for the main channel is considered using the AS-MAC algorithm. In order to prevent the two new nodes from having the same scheduling in the network, before sending the first hello message, the new node waits for a random time about the large size of the competition window. If the new node loses its competition, it will try again to get a new scheduling. The proposed algorithm inherits the inefficiency of the publication from the AS-MAC algorithm. In the proposed algorithm, for packet release operations, each node sends the packet only to its neighbours. Because the neighbouring nodes do not have the same period of awakening and main channel. The proposed algorithm performs neighbour identification in order to maintain the network dynamics. The node listens to each channel periodically so that it can receive a hello message and store its neighbour's scheduling in its neighbour's table and send its scheduling information to its new neighbours by sending hello message. The detection of neighbour's interrupt is determined by the network topology change rate. As soon as the network topology changes, network interruption will decrease.

Many wireless sensor networks are stationary. Due to the fact that wireless sensor networks generally work in the industrial, scientific and medical (ISM) band, the channels and the sleep period, in the initial initialization phase, may later be occupied by other networks. In the proposed method, to avoid this problem, the main channel does not use the same main channel and awakening during the lifetime, but the channel that is least used is selected and the proposed method finds less awaking interruption and then creates a new timing and sends its scheduling information to all neighbors. This operation can be carried out in the neighborhood identification phase. The interrupt of neighbor identification and changing the main channel can be separated.

\section{Implementation, structure and network model}

The implementation of the proposed media access control protocol is done in the MATLAB environment and we didn't use any ready-made tools for simulation.

\section{Energy consumption model}

To better evaluate and compare the proposed method, the network structure and energy consumption model of the method are roughly as the same as the ASMAC method. The network structure is a chain of 4 sensor nodes that sink node is located at the end of the chain. The nodes in the chain send the data to the sink with a multi-stage method. Due to the type of timing of the wake up for nodes, it is assumed that the offset of all of the neighboring nodes is not the same, so there is no collision between the neighboring nodes. Table 1 provides a description of the main variables used in simulation.

Table 1 The main variables of simulations

\begin{tabular}{|c|c|c|}
\hline Variable & Description & Value \\
\hline Ldata & Length of data packet & 50 bytes \\
\hline Lsync & Length of SYNC packe & 18 bytes \\
\hline Lhello & Length of hello packet & 18 bytes \\
\hline Ptx & $\begin{array}{l}\text { Power in data transfer } \\
\text { mode }\end{array}$ & $52.2 \mathrm{~mW}$ \\
\hline Prx & $\begin{array}{l}\text { Power in data receive } \\
\text { mode }\end{array}$ & $56.4 \mathrm{~mW}$ \\
\hline Plisten & Power in listening mode & $56.4 \mathrm{~mW}$ \\
\hline Ps & Power in sleeping mode & $0.003 \mathrm{~mW}$ \\
\hline Plpl & Power in LPL mode & $12.3 \mathrm{~mW}$ \\
\hline tlple & LPL duration & $2.5 \mathrm{~ms}$ \\
\hline $\mathrm{tB}$ & $\begin{array}{l}\mathrm{TX} / \mathrm{RX} \text { duration for a } \\
\text { byte }\end{array}$ & $0.032 \mathrm{~ms}$ \\
\hline tslot & $\begin{array}{l}\text { Duration of the } \\
\text { competition slot }\end{array}$ & $0.04 \mathrm{~ms}$ \\
\hline Scw & $\begin{array}{l}\text { Competition window } \\
\text { size }\end{array}$ & 16 \\
\hline
\end{tabular}

Energy consumption per second is displayed with E, which includes energy consumption related to transfer, receiving, listening, sleeping and LPL that are shown with $\mathrm{E}_{\mathrm{tx}}, \mathrm{E}_{\mathrm{rx}}, \mathrm{E}_{\mathrm{lx}}, \mathrm{E}_{\mathrm{s}}$ and $\mathrm{E}_{\mathrm{LPL}}$, respectively. The general relation of energy is shown in equations (2) to (4), in which $\mathrm{T}$ is the ratio of the time of each section and the $\mathrm{P}$ indicates the power consumption in each section. Sleep time is the time that node does nothing.

$E=E_{t x}+E_{r x}+E_{l x}+E_{l p l}+E_{s}$

$E=T_{t x} P_{t x}+T_{r x} P_{r x}+T_{l x} P_{l x}+T_{l p l} P_{l p l}+T_{s} P_{s}$

$T_{s}=1-T_{t x}+T_{r x}+T_{l x}+T_{l p l}$

In the proposed method, at a time interval that is said to be the time interval of hello or $I_{\text {hello, a hello }}$ message is sent in the network for awareness of the rest of the nodes. In addition, there is a time interval to wake up nodes which is called $\mathrm{I}_{\text {wakeup. }}$ We assume that $\mathrm{I}_{\text {hello, }}$, is $\mathrm{m}$ times of $\mathrm{I}_{\text {wakeup }}$, ie $\mathrm{I}_{\text {hello }}=\mathrm{m} \mathrm{I}_{\text {wakeup }}$. In 
fact, it means that a node receives hello message of its neighbors for each $\mathrm{m}$ packets of data.

\section{Data transfer time}

The data transfer time is calculated from equation (5), in which $\mathrm{T}_{\text {txhello }}, \mathrm{T}_{\text {txdata }}$, and $\mathrm{T}_{\text {txpre }}$ are the ratio of the required times for the hello packet, the main packet of data, and the prerequisites. At each time interval, the hello packet is sent once, so the amount of its required time is calculated from equation (6). The nodes send the obtained data at each time interval, i.e. $\mathrm{I}_{\mathrm{data}}$. Therefore, the time of sending the main data is calculated from relation (7), in which the $\mathrm{N}_{\text {decendants }}$ is equal to the number of nodes of the child for each node. When a node wants to send data, for $\mathrm{m}-1$ wakeup time, only a hello message is sent. Because the hello message does not require a prerequisite, so that from the m-time interval $\mathrm{m}-1$ interval needs to send the prerequisites, so $\mathrm{T}_{\text {txpre }}$ is calculated from equation (8).

$$
\begin{aligned}
& T_{\text {tx }}=T_{\text {txhello }}+T_{\text {txdata }}+T_{\text {txpre }} \\
& T_{\text {txhello }}=\frac{1}{I_{\text {hello }}} L_{\text {hello }} t_{B} \\
& T_{\text {txdata }}=\frac{n_{\text {decendants }}+1}{I_{\text {data }}} L_{\text {data }} t_{B} \\
& T_{\text {txpre }}=\frac{m-1}{m} \frac{n_{\text {decendants }}+1}{I_{\text {data }}} T_{\text {pretx }}
\end{aligned}
$$

\section{Data receive time}

The required time for obtaining information is calculated from equation (9). The nodes receive a hello packet for each $m$ packet they send, so $\mathrm{T}_{\text {rxhello }}$ is calculated from equation (10). Each node receives data packets by its children at any interval of $\mathrm{I}_{\text {data }}$, which is calculated from equation (11). $\mathrm{T}_{\text {rxpre }}$, which is as same as $\mathrm{T}_{\text {txpre }}$ is calculated from equation (12).

$$
\begin{aligned}
& T_{r x}=T_{\text {rxhello }}+T_{\text {rxdata }}+T_{\text {rxpre }} \\
& T_{\text {rxhello }}=\frac{1}{m} \frac{n_{\text {decendants }}+1}{I_{\text {data }}} L_{\text {hello }} t_{B} \\
& T_{\text {rxdata }}=\frac{n_{\text {decendants }}}{I_{\text {data }}} L_{\text {data }} t_{B} \\
& T_{\text {rxpre }}=\frac{m-1}{m} \frac{n_{\text {decendants }}}{I_{\text {data }}} T_{\text {prerx }}
\end{aligned}
$$

\section{Listening time}

The required time for listening is calculated from equation (13) in which $\mathrm{T}_{\text {lxgt }}, \mathrm{T}_{\mathrm{lxcw}}$, and $\mathrm{T}_{\text {lxto }}$ are the wasted time on the clock, the competition time, and the waiting time for completion. The wasted time to adjust and select the channel is calculated from 196 equation (14). When a node sends a data packet, a time is spent to prevent collisions, which is called competition time, its value is calculated from relation (15) in which $S_{c w}$ is the window size of competition. When the nodes wake up, after sending a hello packet, they listen to the channels for half of the time of $t_{0}$. In order to receive possible packets from other nodes, its relation is calculated by equation (16), at which time $t_{0}$ is greater than the maximum time of competition. Its value is calculated from equation (17).

$$
\begin{aligned}
& T_{\text {lx }}=T_{\text {lxgt }}+T_{\text {lxcw }}+T_{\text {lxto }} \\
& T_{\text {lxgt }}=\frac{4 \times r_{\text {clk }}}{2}+\frac{1}{m} \frac{n_{\text {decendants }}+1}{I_{\text {data }}} t_{\text {lple }} \\
& T_{\text {lxcw }}=\frac{n_{\text {decendants }}+1}{I_{\text {data }}} S_{c w} t_{\text {slot }} \\
& T_{\text {lxto }}=\frac{1}{2 I_{\text {hello }}} t_{O} \\
& t_{O}=\left(S_{\text {mcwas }}+1\right) t_{\text {slot }}
\end{aligned}
$$

\section{LPL time}

LPL is a listening method with minimal energy consumption and the minimum time. All nodes need it after waking up and before sending data. Its duration is calculated from equation (18).

$T_{\text {lpl }}=\frac{1}{I_{\text {wakeup }}} t_{\text {lple }}$

\section{Evaluation of proposed method}

The evaluation of the proposed method takes place in two stages. In the first step, the goal is to transfer the one-step nodes, and most of the protocol's capabilities are considered on two nodes that are directly connected, and in the next step, the multimode transmission is considered, and the network structure and number of nodes increase. In simulation, all nodes have an equal wake-up time interval, and each node can communicate only with its neighboring nodes. Also, the default routing method for routing is used in the first step. The time interval of data generation is constant and equal to 100 seconds. But the time interval of wake up and help is optimally calculated with regard to energy consumption. The evaluation of the proposed method is based on the criteria of energy consumption, throughput, delay and the ratio of delivered packets. So we use the wakeup interval, the data generation interval, duty cycle, and traffic load parameters. The experiments in the first stage are carried out with four sensor nodes, which are connected to each other in a chained structure, and the sink node is located at one 
end of the chain. In our implementation, the node number zero is the sink node.

\section{Energy consumption}

Figure $4 a$ shows the amount of packets received. As can be seen, the amount of energy consumed by the proposed method at high speeds of data generation

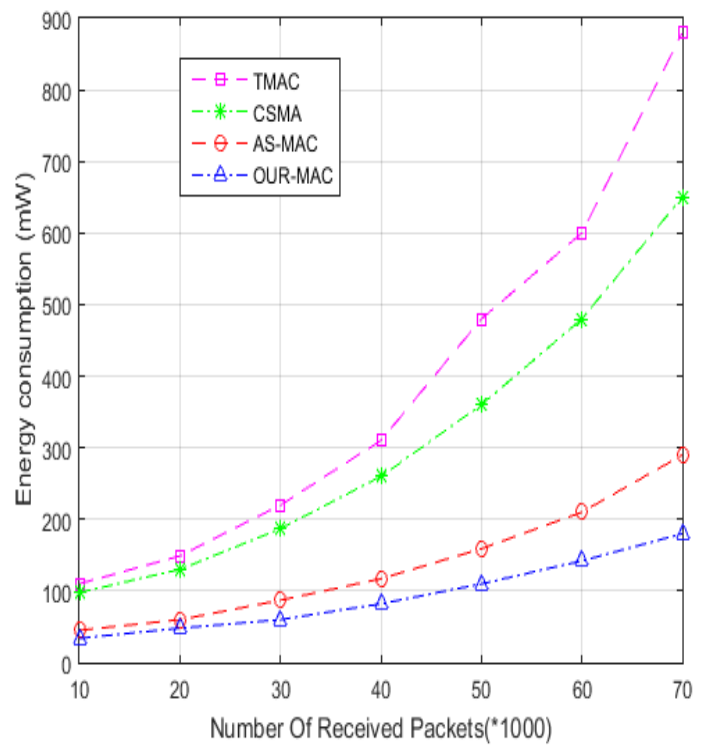

a) Number of received packets

Figure 4 Energy consumption

\section{Other criteria}

The results of packet delivery rate, performance evaluation, the correct packet delivery rate, and

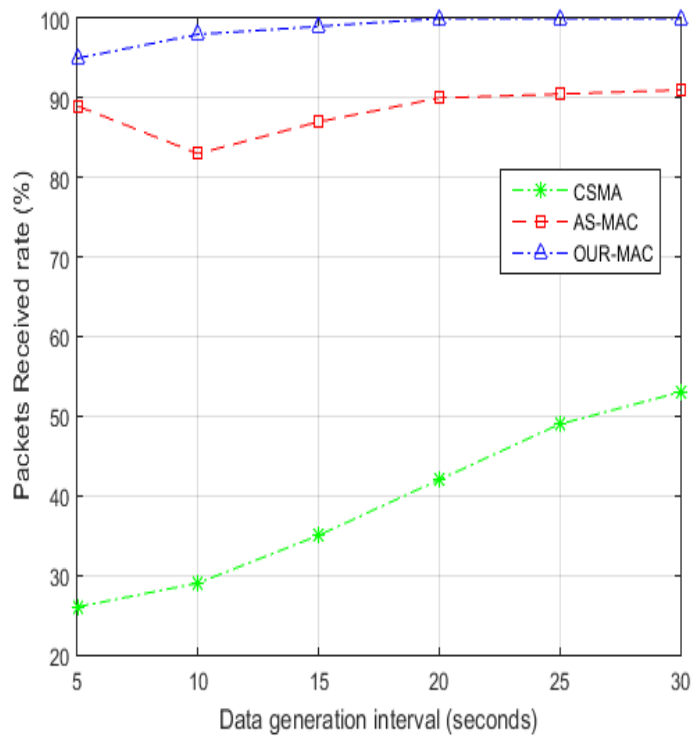

(a) Packets received rate and different number of received packets is lower than the other methods. Figure $4 b$ shows the results of comparing the energy consumption of a node in the proposed method with other methods in different time intervals.

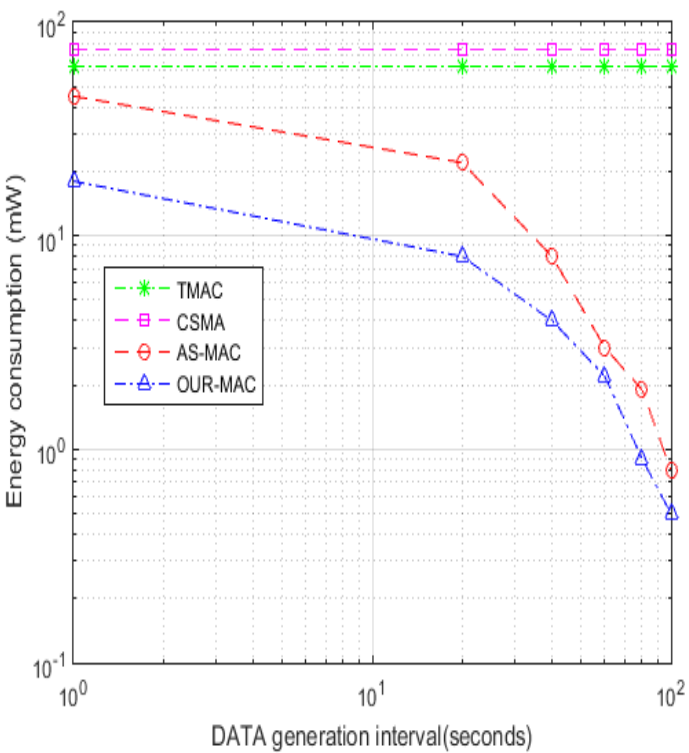

b) Data generation interval

latency in sending multi hop data are shown in Figure 5 (a) to $5(d)$.

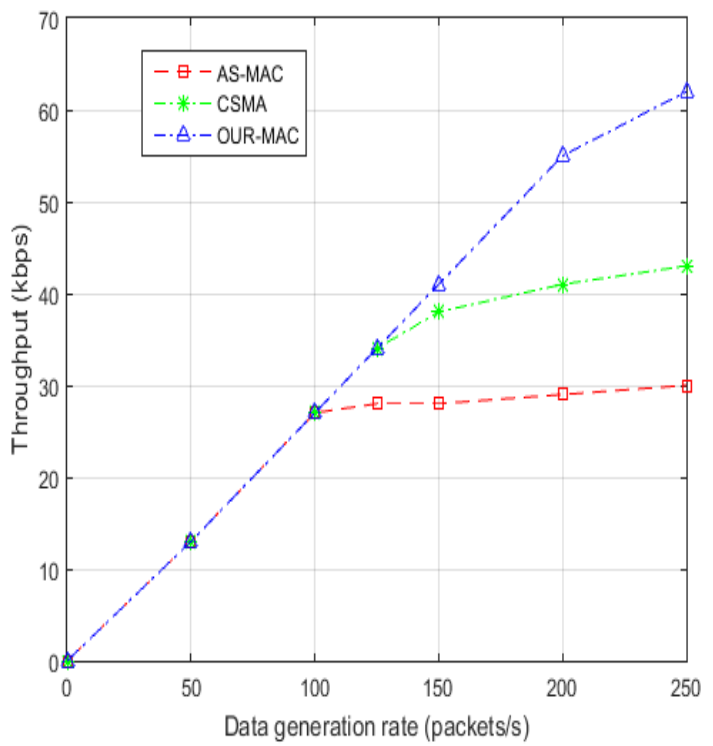

b) Throughput 


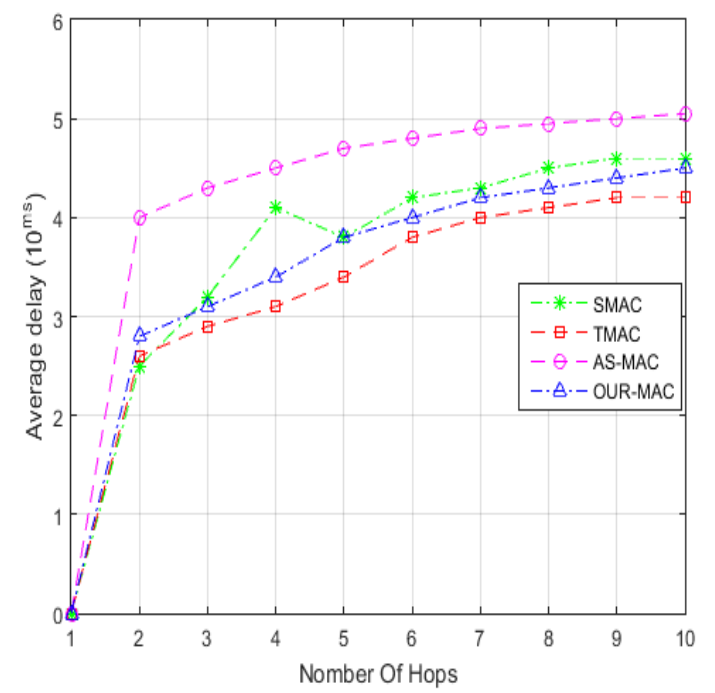

c) Average delay rate in multi hop sending

Figure 5 Comparison of other criteria

The proposed protocol is noncompetitive media access protocols. It is an improved protocol based on AS-MAC. It is in an agreeable manner and asynchronous. It uses the setup parameters like sleep time and awakening nodes and also the meeting nodes with each other in order to exchange data. Proposed protocol has no overhead synchronization. It makes possible for you to use multiple channels to send and receive frames.

A comparison between our proposed method with other MAC-based methods show that our MAC procedure works much better in some cases like energy consumption, operational power, delay and the ratio of packets to the destination, rate packet forwarding, send multiple data steps, eliminating overhead caused by synchronization of nodes, flexibility and efficiency in high traffic. In our proposed method, we also have other parameters such as wake up time efficiency, production time interval, task cycle and traffic load to increase efficiency.

\section{Conclusion}

The main goal of most MACs is to reduce energy consumption. In this paper, after evaluating the MAC protocol, in order to reduce the energy consumption of nodes, a multi-channel media access control protocol is provided. It adjusts the sleeping and awakening of the nodes asynchronously, as well as meeting the nodes in order to exchange data. The use of multiple channels in this way reduces the collision

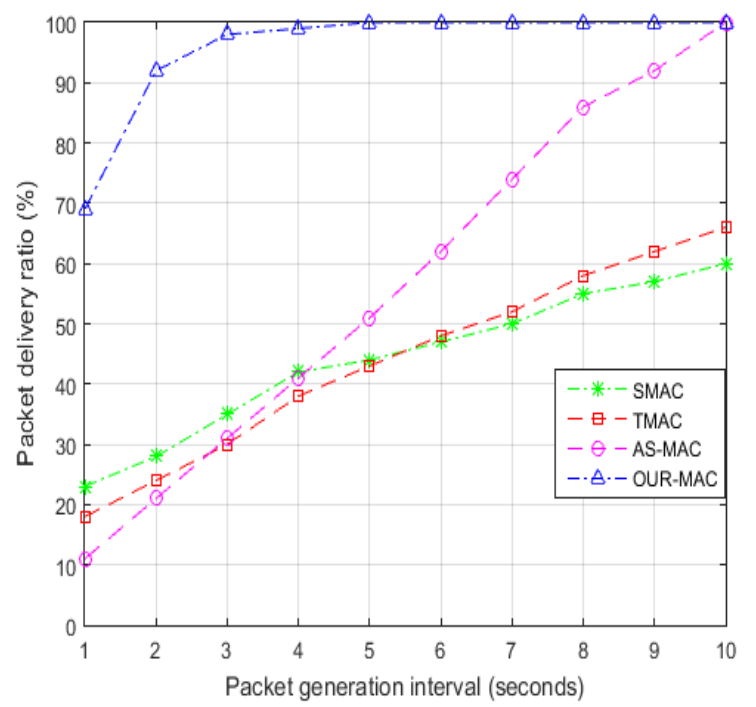

d) Packet delivery rate in multi hop sending

and allows simultaneous transfers in the area of collision nodes. As a result, it increases the efficiency and increase the rate of sending packets in the network. The proposed method has been able to send more data packets in exchange for a specific amount of energy.

\section{Acknowledgment}

None.

\section{Conflicts of interest}

The authors have no conflicts of interest to declare.

\section{References}

[1] Yu Q, Li G, Hang X, Fu K. An energy efficient MAC protocol for wireless passive sensor networks. Future Internet. 2017; 9(2):1-12.

[2] Bagherizadeh M, Kazemi R. A high performance and secure way to time synchronization in wireless sensor network. Journal of Advances in Computer Research. 2016.

[3] Hoang AT, Motani M. Collaborative broadcasting and compression in cluster-based wireless sensor networks. ACM Transactions on Sensor Networks. 2007; 3(3): 1-34.

[4] Enz CC, El-Hoiydi A, Decotignie JD, Peiris V. Wise NET: an ultralow-power wireless sensor network solution. Computer. 2004; 37(8):62-70.

[5] Tang L, Sun Y, Gurewitz O, Johnson DB. EM-MAC: a dynamic multichannel energy-efficient MAC protocol for wireless sensor networks. In proceedings of the international symposium on mobile Ad Hoc networking and computing 2011(p. 23). ACM.

[6] Lim JB, Jang B, Sichitiu ML. MCAS-MAC: a multichannel asynchronous scheduled MAC protocol 
for wireless sensor networks. Computer Communications. 2015; 56:98-107.

[7] Huang P, Xiao L, Soltani S, Mutka MW, Xi N. The evolution of MAC protocols in wireless sensor networks: a survey. IEEE Communications Surveys \& Tutorials. 2013; 15(1):101-20.

[8] Jung WS, Lim KW, Ko YB, Park SJ. Efficient clustering-based data aggregation techniques for wireless sensor networks. Wireless Networks. 2011; 17(5):1387-400.

[9] Verma A, Singh MP, Singh JP, Kumar P. Survey of MAC protocol for wireless sensor networks. In second international conference on advances in computing and communication engineering 2015 (pp. 92-7). IEEE.

[10] Ye W, Heidemann J, Estrin D. An energy-efficient MAC protocol for wireless sensor networks. In proceedings of the annual joint conference of the IEEE computer and communications societies 2002 (pp. 1567-76). IEEE.

[11] El-Hoiydi A, Decotignie JD. WiseMAC: an ultra-low power MAC protocol for multi-hop wireless sensor networks. International symposium on algorithms and experiments for sensor systems, wireless networks and distributed robotics 2004 (pp.18-31). Springer-Verlag Berlin Heidelberg.

[12] El-Hoiydi A. Spatial TDMA and CSMA with preamble sampling for low power Ad Hoc wireless sensor networks. In international symposium on computers and communications 2002 (pp. 685-92). IEEE.

[13] Van Dam T, Langendoen K. An adaptive energyefficient MAC protocol for wireless sensor networks. In proceedings of the international conference on embedded networked sensor systems 2003 (pp. 17180). ACM.

[14] Lu G, Krishnamachari B, Raghavendra CS. An adaptive energy-efficient and low-latency MAC for data gathering in wireless sensor networks. In proceedings of the international symposium on parallel and distributed processing 2004 (p. 224). IEEE.
[15] Lin P, Qiao C, Wang X. Medium access control with a dynamic duty cycle for sensor networks. In wireless communications and networking conference 2004 (pp. 1534-9). IEEE.

[16] Yessad S, Nait-Abdesselam F, Taleb T, Bensaou B. RMAC: reservation medium access control protocol for wireless sensor networks. In local computer networks 2007 (pp. 719-24). IEEE.

[17] Gong D, Zhao M, Yang Y. A multi-channel cooperative MIMO MAC protocol for clustered wireless sensor networks. Journal of Parallel and Distributed Computing. 2014; 74(11):3098-114.

[18] Jang B, Lim JB, Sichitiu ML. An asynchronous scheduled MAC protocol for wireless sensor networks. Computer Networks. 2013; 57(1):85-98.

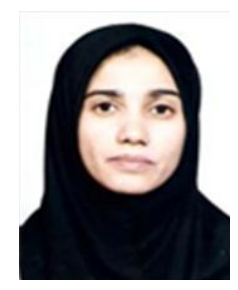

Mahboobeh Irandegani has obtained her BS degree in Computer Software Engineering from Islamic Azad University of Kahnooj branch. She completed her MS degree in IT Network Orientation from Islamic Azad university of Rafsanjan branch. She is a lecturer at Islamic Azad University in Iranshahr branch.

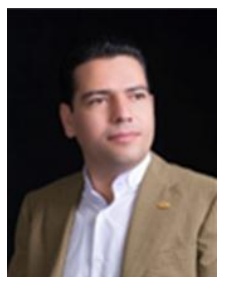

Mehdi Bagherizadeh received his B.Sc. degree from Shahid Bahonar University in Computer Hardware Engineering. He also received M.Sc. and Ph.D degree in Computer Architecture from Science and Research Branch, Islamic Azad University, Tehran, Iran. $\mathrm{He}$ is currently an Assistant Professor in Department of Computer Engineering, Islamic Azad University, Rafsanjan, Iran. He is working on VLSI and circuit design based on CNFET, Application Specific Instruction set Processor, and WSN, and VANET.

Email: m.bagherizadeh@srbiau.ac.ir 